\title{
Self-Consistent Born Approximation for the Hole Motion in the Three-Band Model: a Comparison with Photoemission Experiments
}

\author{
Oleg A. Starykh国, Oziel F. de Alcantara Bonfim, George F. Reiter \\ Texas Center for Superconductivity and Physics Department, University of Houston, Houston, \\ TX 77204-5932
}

(June 14, 1995)

\begin{abstract}
The dispersion relation of the single hole in $\mathrm{CuO}_{2}$ plane is calculated in the self-consistent Born approximation for the thee-band Hamiltonian. We find that direct oxygen-oxygen hopping removes the strong anisotropy of the hole spectrum around the band minima. Our results compare well with recent photoemission measurements of single hole dispersion relation in the $\mathrm{Sr}_{2} \mathrm{CuO}_{2} \mathrm{Cl}_{2}$.
\end{abstract}

PACS: 71.25.-s, 71.27.+a, 79.60.Bm

Typeset using REVTEX 
Recent angle resolved photoemission measurements on different copper-oxide materials [1] have found a number of interesting features in the quasiparticle dispersion. The most interesting is the finding of an almost dispersionless (flat) regions around $(\pi, 0)$ and $(0, \pi)$ points of the Brilloin zone [2]. These flat bands were observed in doped $\mathrm{Bi} 2212$ and $\mathrm{YBaCuO}$ compounds. Recently, APRES experiment was performed on $\mathrm{Sr}_{2} \mathrm{CuO}_{2} \mathrm{Cl}_{2}$ [3], which is an insulating layered cooper oxide. The experiment has the virtue of measuring a single hole dispersion relation in a quantum antiferromagnet. It was found that the overall bandwidth $(\sim 2.2 J)$ and the position of the minimum of the hole band at $(\pi / 2, \pi / 2)$ agree with the $t-J$ model predictions 四. These observations leave no doubts that we are dealing with a hole coupled strongly to the antiferromagnetically interacting localized spins.

At the same time some details of the experimentally found spectrum disagree with these of the $t-J$ model. First of all, dispersion along the magnetic Brilloin zone boundary $((0, \pi) \rightarrow(\pi, 0)$ line $)$ is as strong as along $(0,0) \rightarrow(\pi, \pi)$ line. Second, it turns out that dispersion along $(0,0) \rightarrow(\pi, 0)$ line is very weak, i.e. the band is almost flat, and the hole's energies at $(0,0)$ and $(\pi, 0)$ points were found to coincide within the experimental error [3].

To understand these experimental results we study the motion of a single hole in the three-band model of $\mathrm{CuO}_{2}$ plane. The Hamiltonian of the model is [5]

$$
\begin{aligned}
H= & J \sum_{m, g} \vec{S}_{m} \vec{S}_{m+2 g}+2\left(t_{a}+t_{b}\right) \sum_{\left(m, g, g^{\prime}\right)} \sum_{\sigma, \sigma^{\prime}} \vec{S}_{m} a_{m, g, \sigma}^{+} \vec{s}_{\sigma, \sigma^{\prime}} a_{m, g^{\prime}, \sigma^{\prime}} \\
& +\frac{t_{b}-t_{a}}{2} \sum_{\left(m, g, g^{\prime}\right)} \sum_{\sigma} a_{m, g, \sigma}^{+} a_{m, g^{\prime}, \sigma}-\left|t_{p p}\right| \sum_{\left(m, m^{\prime}, g, g^{\prime}\right)} \sum_{\sigma}\left(a_{m, g, \sigma}^{+} a_{m^{\prime}, g^{\prime}, \sigma}+H . c .\right)
\end{aligned}
$$

Fermion operators $a$ are defined on the oxygen sites, $\vec{s}_{\sigma, \sigma^{\prime}}=\frac{1}{2} \vec{\sigma}_{\sigma, \sigma^{\prime}}, \vec{S}_{m}$ is a localized copper $S=1 / 2$ spin, $\sum_{\left(m, g, g^{\prime}\right)}$ represents independent sum over the four oxygen sites surrounding cooper site $m$, while $\sum_{\left(m, m^{\prime}, g, g^{\prime}\right)}$ means sum over nearest neighbors on the oxygen lattice. Effective hoppings $t_{a}$ and $t_{b}$ are due to the hybridization $t_{p d}$ between $\mathrm{O}$ and $\mathrm{Cu}$ orbitals in the original model [5], whereas $t_{p p}$ is an independent microscopic parameter.

With the help of the canonical transformation

$$
b_{k, \sigma}=\frac{1}{\rho_{k}}\left(\left(1+e^{i k_{x}}\right) a_{k, x, \sigma}+\left(1+e^{i k_{y}}\right) a_{k, y, \sigma}\right)
$$




$$
\begin{aligned}
& d_{k, \sigma}=\frac{1}{\rho_{k}}\left(e^{i k_{x} / 2} \cos k_{y} / 2 a_{k, x, \sigma}-e^{i k_{y} / 2} \cos k_{x} / 2 a_{k, y, \sigma}\right) \\
& a_{k, x, \sigma}=\frac{1}{\sqrt{N}} \sum_{m} a_{m, g_{x}, \sigma} e^{i \vec{k} \vec{r}_{m}}, \quad \rho_{k}=2 \sqrt{1+\gamma_{k}}, \quad \gamma_{k}=\frac{1}{2}\left(\cos k_{x}+\cos k_{y}\right)
\end{aligned}
$$

The hole's part of the Hamiltonian may be rewritten as

$$
\begin{aligned}
H= & \frac{2\left(t_{a}+t_{b}\right)}{N} \sum_{k, k^{\prime}, \sigma, \sigma^{\prime}} \rho_{k} \rho_{k^{\prime}} b_{k, \sigma^{\prime}}^{+} b_{k^{\prime}, \sigma} \vec{s}_{\sigma, \sigma^{\prime}} \vec{S}_{k-k^{\prime}}+\frac{t_{b}-t_{a}}{2} \sum_{k, \sigma} \rho_{k}^{2} b_{k, \sigma}^{+} b_{k, \sigma} \\
& -4\left|t_{p p}\right| \sum_{k, \sigma}\left(f_{1}(k)\left(b_{k, \sigma}^{+} b_{k, \sigma}-d_{k, \sigma}^{+} d_{k, \sigma}\right)+f_{2}(k)\left(d_{k, \sigma}^{+} b_{k, \sigma}+b_{k, \sigma}^{+} d_{k, \sigma}\right)\right),
\end{aligned}
$$

where we introduced short-hand notations for

$$
\begin{aligned}
& f_{1}(k)=\frac{\left(1+\cos k_{x}\right)\left(1+\cos k_{y}\right)}{\rho_{k}^{2}} \\
& f_{2}(k)=\frac{\left(\cos k_{y}-\cos k_{x}\right) \cos k_{x} / 2 \cos k_{y} / 2}{\rho_{k}^{2}}
\end{aligned}
$$

Note that spin-fermion interaction term couples $C u$ spins only to the symmetric combination of oxygen fermion operators, i.e. to the $b$-operators. Another, $d$-band, appears only in direct $O-O$ hopping.

We will treat the copper spins within the spin wave approximation [6]

$$
\begin{aligned}
& S_{m}^{ \pm}=S\left(1 \pm e^{i \vec{q}_{0} \vec{r}_{m}}\right) \alpha_{m}+S\left(1 \mp e^{i \vec{q}_{0} \vec{r}_{m}}\right) \alpha_{m}^{+}, \\
& S_{m}^{z}=e^{i{\overrightarrow{q_{0}}}_{m}}\left(S-\alpha_{m}^{+} \alpha_{m}\right)
\end{aligned}
$$

Magnon operators are defined in the whole original Brilloin zone, and $q_{0} \equiv(\pi, \pi)$. The spin Hamiltonian is diagonalized then by the standard Bogoliubov transformation

$$
\begin{aligned}
& \left(\begin{array}{c}
\alpha_{-q} \\
\alpha_{q}^{+}
\end{array}\right)=\left(\begin{array}{cc}
u_{q} & v_{q} \\
v_{q} & u_{q}
\end{array}\right)\left(\begin{array}{c}
\beta_{-q} \\
\beta_{q}^{+}
\end{array}\right), \\
& u_{q}=\sqrt{\frac{1+\omega_{q}}{2 \omega_{q}}}, \quad v_{q}=-\operatorname{sgn}\left(\gamma_{q}\right) \sqrt{\frac{1-\omega_{q}}{2 \omega_{q}}}, \quad \omega_{q}=4 J S \sqrt{1-\gamma_{q}^{2}}
\end{aligned}
$$

It is convenient, following [6], to introduce staggered fermion operators as well

$$
\begin{aligned}
& b_{k, \sigma}^{+}=\frac{1}{2} \sum_{s}\left(c_{k, s}^{+}+4 \sigma s c_{k+q_{0}, s}^{+}\right) \\
& d_{k, \sigma}^{+}=\frac{1}{2} \sum_{s}\left(z_{k, s}^{+}+4 \sigma s z_{k+q_{0}, s}^{+}\right), \quad s, \sigma= \pm \frac{1}{2}
\end{aligned}
$$


After somewhat lengthly algebra we find that $H=H_{0}+H_{\text {int }}$, where the free part is

$$
\begin{aligned}
H_{0}= & \sum_{q} \omega_{q} \beta_{q}^{+} \beta_{q}+\sum_{k, \sigma, \sigma^{\prime}} \epsilon_{k} \sigma_{\sigma, \sigma^{\prime}}^{z} c_{k, \sigma}^{+} c_{k, \sigma^{\prime}} \\
& +2\left(t_{b}-t_{a}\right) \sum_{k, \sigma}\left(c_{k, \sigma}^{+} c_{k, \sigma}+\gamma_{k} c_{k, \sigma}^{+} c_{k,-\sigma}\right)+H_{p p}
\end{aligned}
$$

Here $\epsilon_{k}=\frac{t_{a}+t_{b}}{4} \rho_{k} \rho_{k+q_{0}}$ is a free dispersion due to the $\sigma^{z} S^{z}$ coupling in the Hamiltonian (3). Note that free dispersion term is absent in the $t-J$ model. In the new basis direct oxygen-oxygen hopping looks a bit complicated

$$
\begin{aligned}
H_{p p}= & -4\left|t_{p p}\right| \sum_{k}\left(f_{1}(k) \sum_{\sigma=+,-}\left(c_{k, \sigma}^{+} c_{k, \sigma}-z_{k, \sigma}^{+} z_{k, \sigma}\right)\right. \\
& +f_{1}\left(k+q_{0}\right)\left(\left(c_{k,+}^{+}-c_{k,-}^{+}\right)\left(c_{k,+}-c_{k,-}\right)-\left(z_{k,+}^{+}-z_{k,-}^{+}\right)\left(z_{k,+}-z_{k,-}\right)\right) \\
& \left.+\left\{f_{2}(k)\left(z_{k,+}^{+}+z_{k,-}^{+}\right)\left(c_{k,+}+c_{k,-}\right)+f_{2}\left(k+q_{0}\right)\left(z_{k,+}^{+}-z_{k,-}^{+}\right)\left(c_{k,+}-c_{k,-}\right)+\text { H.c. }\right\}\right),
\end{aligned}
$$

where $+(-)$ subscript denotes $+\frac{1}{2}\left(-\frac{1}{2}\right)$ spin projection.

The advantage of this representation is that interaction part of the Hamiltonian has a simple form

$$
H_{i n t}=\frac{1}{\sqrt{N}} \sum_{k, q, \sigma, \sigma^{\prime}} U_{\sigma, \sigma^{\prime}}(k, q)\left(c_{k-q, \sigma}^{+} c_{k, \sigma^{\prime}} \beta_{q}^{+}+c_{k, \sigma^{\prime}}^{+} c_{k-q, \sigma} \beta_{q}\right)
$$

For completeness we give an explicit form of the interaction vertex $U$ here

$$
\begin{gathered}
U(k, q)=\frac{t_{a}+t_{b}}{4}\left(\begin{array}{cc}
u_{q} \rho_{k,-} \rho_{k-q,+}+v_{q} \rho_{k,+} \rho_{k-q,-} & u_{q} \rho_{k,+} \rho_{k-q,+}+v_{q} \rho_{k,-} \rho_{k-q,-} \\
u_{q} \rho_{k,-} \rho_{k-q,-}+v_{q} \rho_{k,+} \rho_{k-q,+} & u_{q} \rho_{k,+} \rho_{k-q,-}+v_{q} \rho_{k,-} \rho_{k-q,+}
\end{array}\right), \\
\rho_{p,-} \equiv \rho_{p}-\rho_{p+q_{0}}, \quad \rho_{p,+} \equiv \rho_{p}+\rho_{p+q_{0}} .
\end{gathered}
$$

To calculate the hole's Green's function

$$
\begin{aligned}
G(k, \omega) & =\left[G_{0}^{-1}(k, \omega)-\Sigma(k, \omega)\right]^{-1}, \\
G_{0}(k, \omega) & =\left(\omega-H_{0}\right)^{-1}
\end{aligned}
$$

we use the self-consistent Born approximation [7]

$$
\Sigma_{\sigma, \sigma^{\prime}}(k, \omega)=\frac{1}{N} \sum_{\nu, \nu^{\prime}} \sum_{q} U_{\nu, \sigma}(k, q) G_{\nu, \nu^{\prime}}\left(k-q, \omega-\omega_{q}\right) U_{\nu^{\prime}, \sigma^{\prime}}(k, q)
$$


In the presence of nonzero oxygen-oxygen hopping $t_{p p}, G(k, \omega)$ is $4 \times 4$ matrix, but the interaction vertices $U_{\nu, \sigma}(k, q)$ are $2 \times 2$ matrices, because there is no coupling between magnons and $z$-fermions.

The self-consistent Born approximation (SCBA) consists in neglecting vertex corrections to the self-energy. It was found to work well for the $t-J$ model [4], and was applied to the three-band model in Refs. [8,9]. It predicts the band minima at $(\pi / 2, \pi / 2)$ and bandwidth proportional to $J[8]$, as does the $t-J$ model. The energy dispersion, in the absence of $t_{p p}$, is very similar in the two models. The main difference is that hole has a spin $1 / 2$. For momenta close to $(\pi / 2, \pi / 2)$ the hole's spin is antialigned with the Copper spin, thus forming a singletlike excitation [8]. However, away from minima a strong mixture of the triplet state appears [8,10].

Omitting $H_{\text {int }}$ for a moment we see that at $t_{p p}=0$ the spectrum has a degenerate minima along the $\gamma_{q}=0$ curve. Turning on $t_{p p}$ lifts the degeneracy, and the minima moves to $(\pi / 2, \pi / 2)$ [10]. At the same time the energy at $k=(0,0)$ coincides with that at $(\pi, 0)$. It is worth noting that the approximate relation $E(\pi / 2,0)=0.5 E(\pi / 2, \pi / 2)$ holds. An analogous one-hole dispersion relation was obtained in [1] by doing small-U perturbation theory of the one-band Hubbard model.

Consider now the effect of interaction. Equation (12) was solved numerically by the simplest iteration procedure. The Green's function at energy $\omega$ on the right hand side of (12) was used to calculate the self-energy at the next energy slice, $\omega+\Delta \omega$. We choose $\Delta \omega$ to be 0.01 in units of $t_{a}+t_{b}$, and introduce the damping constant $\delta=0.01$ to achieve numerical stability. To check for finite size effects, we used an integration with 17 points for the 'best fit' (see below), and found no practical difference with the 13-points integration. To check the validity of the procedure we used it to solve the self-consistent equation for the $t-J$ model, and found good agreement with existing results [4].

Our 'best fit' to the experimental data is shown on Fig.1. Parameters of the fit are the following: $t_{a}=0.19, t_{b}=0.5, t_{p p}=0.76, J=0.125$ in $\mathrm{eV}$. It is quite easy to achieve a reasonable agreement with experiment along $(0,0) \rightarrow(\pi / 2, \pi / 2) \rightarrow(\pi, 0)$ directions. 
The main problem is to fit experiment around the $(\pi / 2,0)$ point. Our search for the 'best fit' mainly consisted in minimizing the deviation between Eq.(12) and experimental data in the region of momenta between $(\pi, 0)$ and $(\pi / 2,0)$. We found that spectrum $E(k)$ is not affected by small variations of $t_{a}$ and $t_{b}$ while keeping their sum fixed. For example, $t_{a}=0.07, t_{b}=0.62, t_{p p}=0.76$ fits the experimental $E(k)$ as well as the 'best fit'. We found that even better agreement along $k_{y}=0$ line can be reached by choosing unrealistical values of parameters: $t_{a}=0.31, t_{b}=0.94, t_{p p}=1.37$. But the bandwidth gets higher for these values (Fig.2).

The spectral density $A(k, \omega)=\frac{1}{\pi} \operatorname{Im}(\operatorname{Tr} G(k, \omega))$ for the 'best fit' is shown on Fig.3. Quasiparticle peaks are observed at all values of $k$. At $k=(0,0)$ the weight of the quasiparticle pole is smallest, most of the weight is concentrated at the very strong peak at $\omega=0$ [9]. To see that lowest peaks of $A(k, \omega)$ indeed represent quasiparticles we analyzed the $\delta$-dependence of peaks at $k=(\pi / 2, \pi / 2),(\pi, 0)$ and $(\pi / 2,0)$. In all cases peak fits well by a

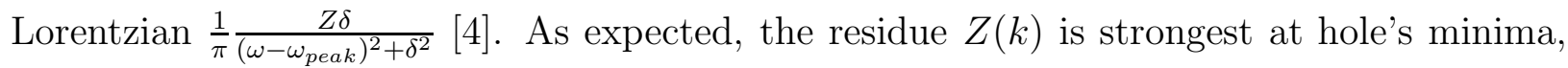
$Z(\pi / 2, \pi / 2) \simeq 0.25$, whereas $Z(\pi / 2,0)$ is 4 times smaller. It is even smaller then that at the $(\pi, 0)$ point $\left(\frac{Z(\pi / 2, \pi / 2)}{Z(\pi, 0)} \simeq 6\right)$ simply because quasiparticle's energy at this momentum is higher then that at the $k=(\pi / 2,0)$. It is worth noticing that our value of $Z(\pi / 2, \pi / 2)$ is quite close to that in the t-J model 画. Smallness of the ratio $\frac{Z(\pi / 2,0)}{Z(\pi / 2, \pi / 2)}$ indicates that experimental determination of the hole's energy around $k=(\pi / 2,0)$ can be difficult.

Recently Nazarenko et al. [12 reported theoretical analysis of the same problem. They showed numerically that the spectrum isotropy around the band minima can be explained within the single-band $t-J$ model with the small value of intrasublattice hopping $t^{\prime} \sim J$. However 'flatness' around $(\pi / 2,0)$ was not explained. Also they (see also [13]) performed calculations of the $E(k)$ based on the variational wavefunction for the three-band model, and reached an impressive agreement with experimental results at all values of $k$. Theoretical reason for such agreement is not clear to us. The only difference with our analysis is that they also included local Kondo - like coupling between $C u$ and $O$ spins $\left(J_{2}\right.$ term in notations of Ref. [10], or $K_{p d}$ in notations of [14]). Notice that such local Kondo-term has no effect 
on the spectrum at the mean-field level, because it is proportional to $\left(\vec{S}_{m}+\vec{S}_{m+2 g}\right) \cdot \vec{\sigma}_{m+g}$, which is zero on the Neel state. On the quantum level this term does affect structure of Eq.(12) significantly, as it couples magnons with both $c$ - and $z$-fermions. We have solved Eq.(12), reformulated to take into account $J_{2}$ coupling, and found no qualitative changes in the $E(k)$.

To make a connection with existing estimates of the microscopical parameters based on the density-functional approach we present on Fig.4 results of our calculations with parameters from Hybertsen et al. [14]. Clearly, the agreement with experiment is reasonable.

To summarize, we found that experimental results of Wells et al. [3] can be reasonably well described within the three-band model with values of microscopical parameters in good agreement with existing estimates. The main result is that direct oxygen-oxygen hopping $t_{p p}$ is the largest parameter in the problem. This is necessary for explaining the isotropy of the spectrum arount the minima at $(\pi / 2, \pi / 2)$. At the same time our results show the presence of the quasiparticle pole in the Green's function at $k=(\pi / 2,0)$ also, whereas experimentally the possibility of no coherent excitations at this value of momentum can not be ruled out. Higher experimental accuracy is necessary to clarify this question.

We thank B. Wells for providing us with experimental data. We also thank A.Chubukov, D.Frenkel, and K.Musaelian for discussions. We are grateful to R.Gooding and K.Vos for sending us their results and useful discussions. 


\section{REFERENCES}

a Present address: Department of Physics, University of California, Davis, CA 95616

b On leave from the Institute for High Pressure Physics, 142092, Troitsk, Moscow Region, Russia

[1] Z.-X.Shen, D.S.Dessau, Phys.Rep. 253, 1 (1995)

[2] D.S.Dessau et al., Phys. Rev. Lett. 71, 2781 (1993); K.Gofron et al., J.Phys.Chem.Solids 54, $1193(1993)$

[3] B.O.Wells et al., Phys. Rev. Lett. 74, 964 (1995)

[4] Z.Liu and E.Manousakis, Phys. Rev. B 45, 2425 (1992)

[5] V.J.Emery and G.Reiter, Phys. Rev. B 38, 4547 (1988); Phys. Rev. B 38, 11938 (1988)

[6] A.Ramsak and P.Prelovsek, Phys. Rev. B 42, 10415 (1990)

[7] C.L.Kane, P.A.Lee and N.Read, Phys. Rev. B 39, 6880 (1989)

[8] O.F. de Alcantara Bonfim and G.Reiter, in Proceedings of the Univ. of Miami Workshop on Electronic Structure and Mechanisms for High Temperature Superconductivity, ed. J.Ashkenazi, New York, Plenum Press, (1991)

[9] V.V.Kabanov and A.Vagov, Phys. Rev. B 47, 12134 (1993)

[10] D.M.Frenkel, R.J.Gooding, B.I.Shraiman, and E.D.Siggia, Phys. Rev. B 41, 350 (1990)

[11] A.V.Chubukov and K.A.Musaelian, J.Phys.:Cond.Matter 7, 1333 (1995)

[12] A.Nazarenko et al., Phys. Rev. B 51, 8676 (1995)

[13] K.J.E.Vos and R.J.Gooding, private communication

[14] M.S.Hybertsen, E.B.Stechel, M.Schluter, and D.R.Jennison, Phys. Rev. B 41, 11068 (1990) 


\section{FIGURES}

FIG. 1. 'Best fit' to the experimentally measured quasiparticle dispersion.

FIG. 2. Example of fit with 'unrealistic' values of parameters.

FIG. 3. Spectral function of hole at different momenta. Parameters correspond to the 'best fit', Fig.1. $\omega$ is in units of $t_{a}+t_{b}$.

FIG. 4. Quasiparticle dispersion with parameters based on density-functional calculations, Ref.[14]. 
Fig.1 of Starykh et al.

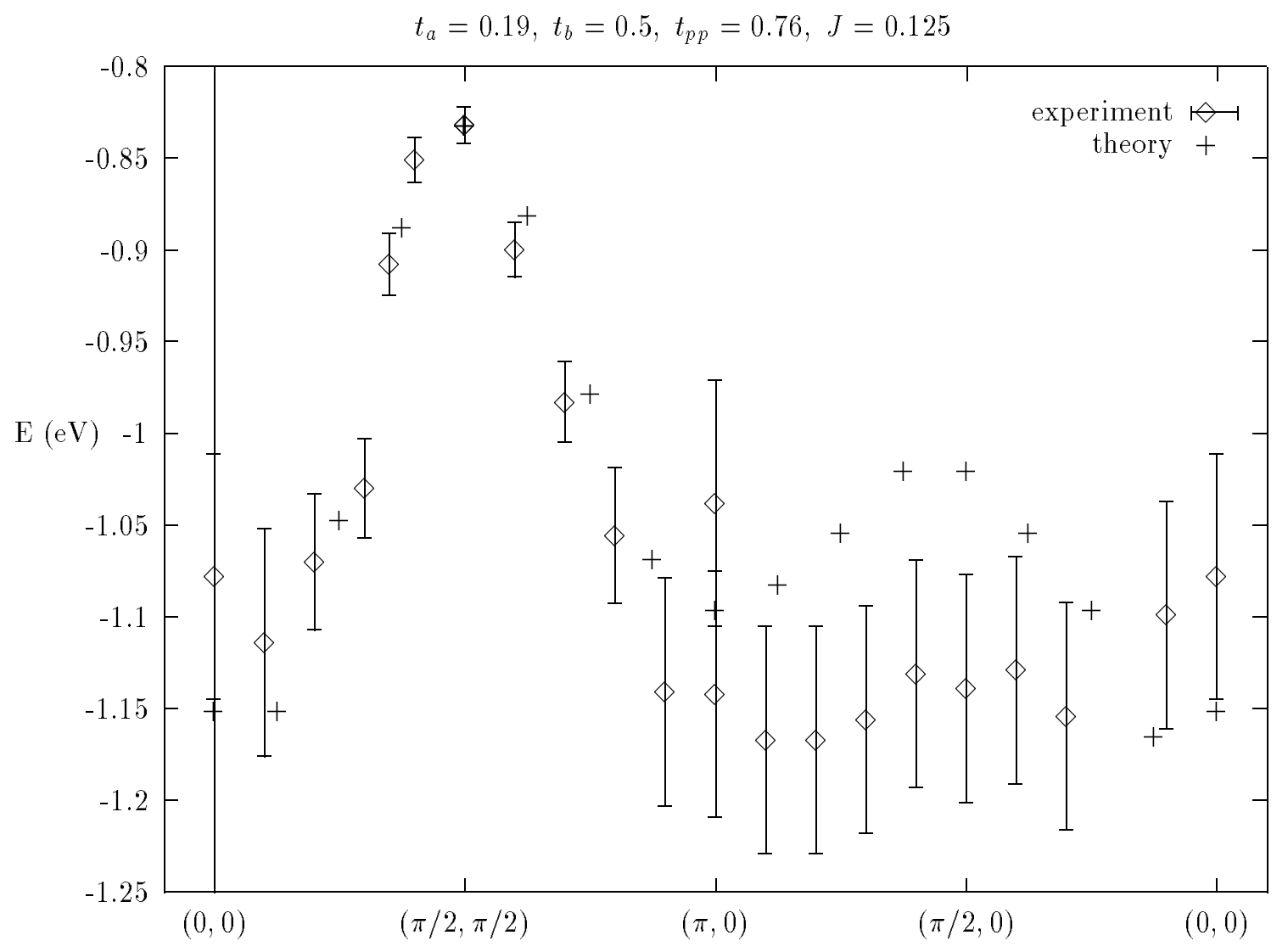


Fig.2 of Starykh et al.

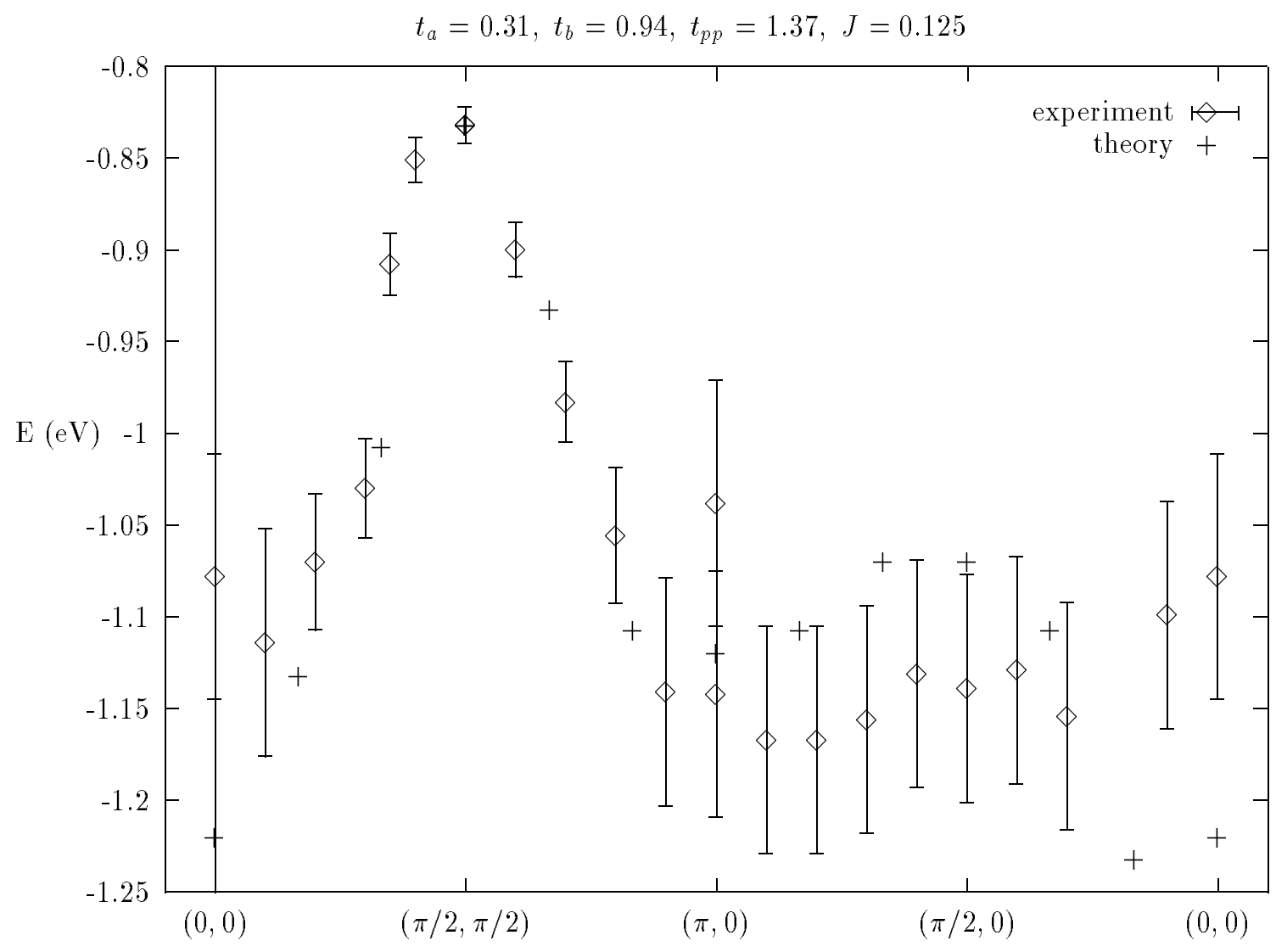


Fig.4 of Starykh et al.

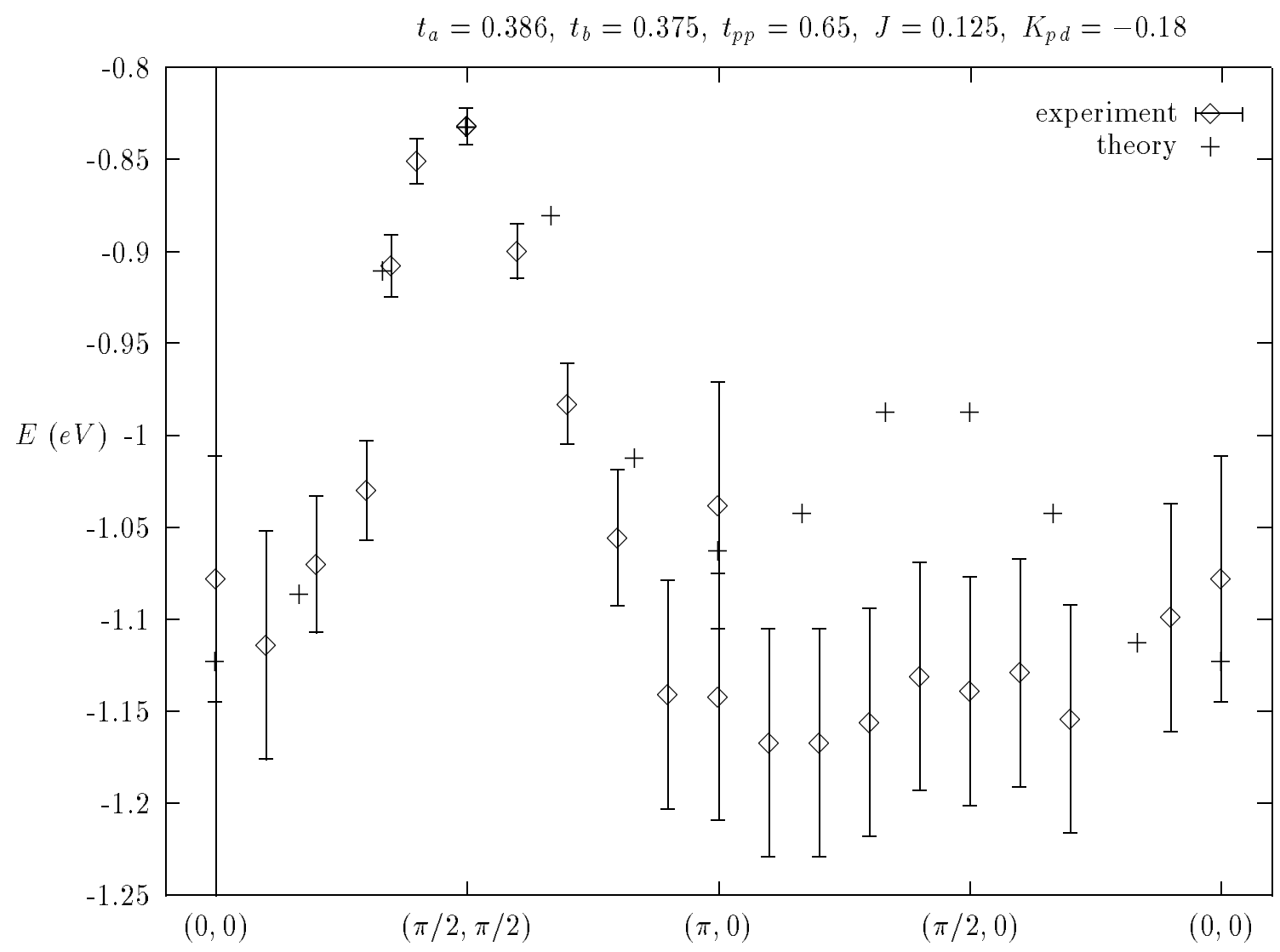


Fig.3 of Starykh et al.
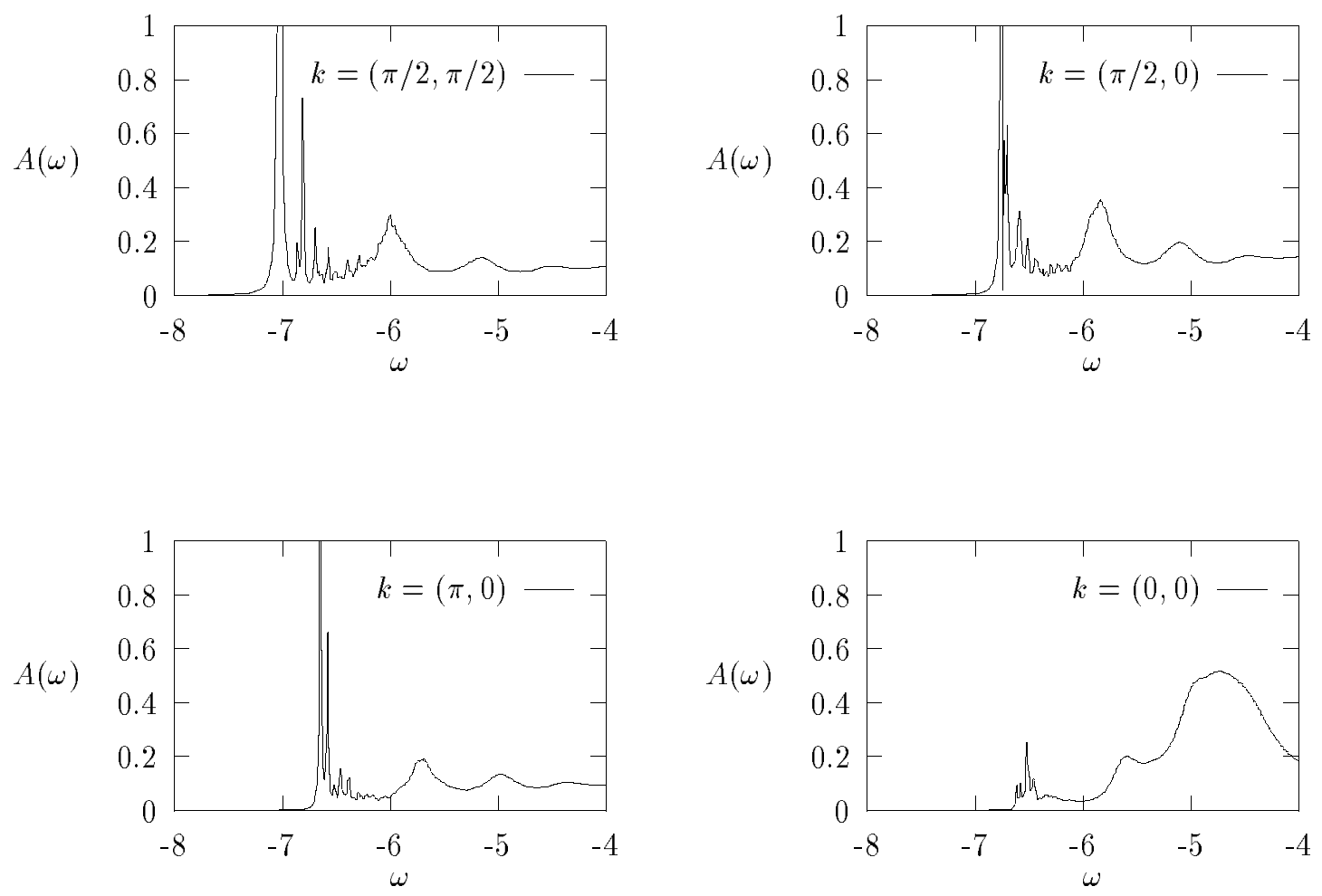